\title{
Adaptabilidade e estabilidade de híbridos de famílias endogâmicas de milho, obtidos pelo método dos híbridos crípticos $^{(1)}$
}

\begin{abstract}
Maria Teresa Gomes Lopes ${ }^{(2)}$, José Marcelo Soriano Viana ${ }^{(3)}$ e Ricardo Lopes ${ }^{(2)}$
Resumo - Neste trabalho avaliou-se o comportamento de híbridos de famílias endogâmicas de milho obtidos pelo método dos híbridos crípticos, produzidos pelo programa de melhoramento de milho do Departamento de Biologia da UFV. A caracterização dos híbridos foi feita com base em 22 ambientes, usando-se os métodos de Eberhart \& Russell e de Carneiro. Pelo método de Eberhart \& Russell, foram identificados 15 híbridos com produção acima da média geral. Entre eles, 53,3\% tiveram bom desempenho em qualquer ambiente, e alta estabilidade (84-6, 86-1, 86-19, 86-11, 86-15, 86-21, 86-27 e 86-10), e $20 \%$ são mais responsivos à melhoria ambiental e possuem alta estabilidade (86-22, 85-2 e 84-5). Os híbridos 85-1, 85-3 e 86-2 apresentaram bom desempenho em qualquer ambiente e baixa estabilidade, correspondem também a $20 \%$ dos mais produtivos. O último (86-8), correspondendo a $6,7 \%$, apresentou adaptação específica a ambientes favoráveis, e baixa estabilidade. Pela análise, considerando o método de Carneiro, foram identificados os híbridos 86-22, 86-8, 84-5, 85-2 e 86-19 como os mais responsivos à melhoria do ambiente. Os híbridos 86-11, 85-3, 86-27 e 86-2 apresentaram adaptação a ambientes desfavoráveis.
\end{abstract}

Termos para indexação: Zea mays, métodos de melhoramento, seleção, adaptação.

Adaptability and stability patterns in endogamic hybrid maize families, obtained by the cryptic hybrids method

\begin{abstract}
The behavior of endogamic hybrid maize families was assessed using the cryptic hybrids method. This work was conduced by the maize breeding program at Departamento de Biologia of the Universidade Federal de Viçosa in Brazil. Twenty-two environments were used to characterize the hybrids using Eberhart \& Russel and Carneiro methods. Using the first method, 15 hybrids were identified with yield higher than the general mean. Among them, $53.3 \%$ had general adaptability and high stability (84-6, 86-1, 86-19, 86-11, 86-15, 86-21, 86-27 and 86-10), and 20\% had specific adaptation to favorable environments and high stability (86-22, 85-2 and 84-5). The hybrids 85-1, 85-3 and 86-2 had general adaptability associated with low stability, and also corresponding to a fraction $20 \%$ of the most productive hybrids. The last hybrid (86-8) corresponded to $6.7 \%$ and had specific adaptation to favorable environments and low stability. From the analysis using Carneiro's method, the hybrids 86-22, 86-8, 84-5, 85-2 and 86-19 were identified as the most adapted to favorable environments. The hybrids $86-11,85-3,86-27$ and 86-2 were adaptable to unfavorable environments.
\end{abstract}

Index terms: Zea mays, breeding methods, selection, adaptation.

(1) Aceito para publicação em 11 de julho de 2000

Extraído da dissertação de mestrado, apresentada pelo primeiro autor à Universidade Federal de Viçosa (UFV), Viçosa, MG. Parcialmente financiado pelo CNPq.

(2)ESALQ, Laboratório de Genética Molecular, Caixa Postal 9, CEP 13418-900 Piracicaba, SP.

E-mail:mtglopes@carpa.ciagri.usp.br,

rlopes@carpa.ciagri.usp.br

(3)UFV, Dep. de Biologia Geral, CEP 36571-000 Viçosa, MG. E-mail: jmsviana@mail.ufv.br

\section{Introdução}

O método dos híbridos crípticos, sugerido por Hallauer (1967) e Lonnquist \& Williams (1967) visa à obtenção de híbridos superiores, com base no aumento da freqüência de genes complementares. Teoricamente, é excelente para obtenção de híbridos de linhagens, uma vez que ao longo de sua execução são previstos cerca de seis ciclos de seleção para ca- 
pacidade específica de combinação. Entretanto, pode ser considerado de uso relativamente restrito, em comparação com o processo normal de obtenção de linhagens e híbridos, no qual também são realizados cerca de seis ciclos de seleção, mas somente no final avalia-se a capacidade específica de combinação.

Por este método, pelo menos uma das plantas utilizadas nos cruzamentos deve ser prolífica, sendo uma das espigas usada para cruzamento interpopulacional, e a outra, para produção de família endogâmica. Os híbridos resultantes do primeiro cruzamento interpopulacional $\left(\mathrm{S}_{0} \times \mathrm{S}_{0}\right)$ são avaliados com base nas características agronômicas de interesse. As sementes autofecundadas de cada planta constituirão a primeira geração de autofecundação $\left(\mathrm{S}_{1}\right)$, para a obtenção das linhagens. Selecionam-se as melhores progênies $S_{1}$ com base nos híbridos $S_{0} x$ $\mathrm{S}_{0}$ superiores, as quais são semeadas em linhas pareadas. Autofecundam-se e cruzam-se novamente os pares de plantas em que pelo menos uma delas seja prolífica. Os cruzamentos $\left(\mathrm{S}_{1} \times \mathrm{S}_{1}\right)$ são avaliados e as progênies $S_{2}$ correspondentes aos híbridos superiores são selecionadas e semeadas novamente em fileiras pareadas. O método recomenda que o procedimento descrito seja repetido por várias gerações de autofecundação, até se atingir um grau de endogamia satisfatório, avaliando-se a cada geração as linhagens obtidas para capacidade específica de combinação.

O método dos híbridos crípticos tem uso limitado em programas de melhoramento, principalmente devido à falta de informações que permitam aos melhoristas avaliar seu potencial, o que, conseqüentemente, impede que o método se torne de uso comum para a obtenção de híbridos. Os híbridos de famílias endogâmicas $\mathrm{S}_{1} \times \mathrm{S}_{1}, \mathrm{~S}_{2} \times \mathrm{S}_{2}, \mathrm{~S}_{3} \times \mathrm{S}_{3}$ e $\mathrm{S}_{4} \times$ $\mathrm{S}_{4}$, normalmente avaliados durante o processo de obtenção de híbridos crípticos, são materiais heterogêneos, por se originarem de famílias com grau variado de heterozigose. As avaliações desses híbridos em diferentes ambientes poderá informar sobre o tipo de comportamento predominante dos híbridos crípticos em face das variações ambientais.

A variação no comportamento de populações em diferentes ambientes está, muitas vezes, relacionada com sua base genética. Em geral, as que possuem maior base genética interagem menos com o am- biente, e, portanto, são mais estáveis (Allard \& Bradshaw, 1964; Allard, 1971; Feaster \& Turcotte, 1973). Em trabalhos realizados com milho, vários autores concluíram que os materiais menos homogêneos (híbridos triplos, híbridos duplos, híbridos intervarietais e variedades) são mais estáveis quanto à produção, comparativamente aos mais homogêneos (Sprague, 1955; Eberhart et al., 1964; Lemos, 1976; Ruschel, 1986). Paterniani (1965) atribuiu essa maior capacidade adaptativa das populações heterogêneas ao grande número de genótipos que as constituem. Borém (1999) afirma que genótipos heterozigóticos são menos sujeitos às influências do ambiente do que os homozigóticos, e que populações heterogêneas possuem maior poder tamponante do que as homogêneas, de forma que uma relativa estabilidade seja mantida. Entretanto, diversos autores encontraram resultados opostos em seus estudos com milho, indicando que materiais mais homogêneos são mais estáveis (Eberhart \& Russell, 1969; Ruschel \& Penteado, 1970; Costa, 1976; Naspolini Filho, 1976).

Existem, na literatura, vários métodos para avaliar o comportamento de cultivares. Eberhart \& Russell (1966) propuseram um modelo de regressão linear simples, que sobressaiu nos estudos de desempenho genotípico. Apesar de alguns autores terem proposto modificações nesse método, observa-se, ainda, um maior uso do procedimento sugerido originalmente. Carneiro (1998), visando ao aprimoramento do método proposto por Lin \& Bins, sugeriu a decomposição da medida de adaptabilidade e estabilidade de comportamento para ambientes favoráveis e desfavoráveis, tornando o método ainda mais eficiente $\mathrm{e}$ promissor no uso pelos melhoristas.

O objetivo deste trabalho foi caracterizar híbridos de famílias endogâmicas de milho, obtidos pelo método dos híbridos crípticos.

\section{Material e Métodos}

Foram usados neste estudo híbridos crípticos de milho obtidos de famílias endogâmicas, derivadas de programas de seleção recorrente recíproca (SRR). Os trabalhos de obtenção e avaliação dos híbridos foram conduzidos pelo Setor de Genética do Departamento de Biologia Geral da Universidade Federal de Viçosa. As famílias "dent" e 
"flint" que deram origem aos híbridos são derivadas de dois programas de SRR, um com compostos produzidos pela recombinação de progênies $S_{2}$ selecionadas, e outro, com compostos produzidos pela recombinação de progênies $\mathrm{S}_{4}$ não selecionadas. A caracterização dos tratamentos utilizados está na Tabela 1. Dos 29 tratamentos avaliados, 23 são híbridos de famílias endogâmicas (tratamentos 1 a 23) e seis são híbridos de compostos (tratamentos 24 a 29). Pelo fato de não haver testemunhas comerciais comuns aos 22 ensaios, foram utilizados como testemunhas, na análise conjunta, os seis híbridos de compostos, os quais foram avaliados em todos os ensaios e apresentaram desempenho estatisticamente igual ou superior ao de testemunhas comerciais, na comparação múltipla de médias na análise da maioria dos ensaios individualmente
(Lopes, 1999). Apesar de as testemunhas não serem materiais comerciais, estas são originadas de programas de SRR com base em irmãos completos, os quais são um bom referencial para os híbridos de interesse, pois as famílias endogâmicas cruzadas para obtê-los também são provenientes de compostos com origem semelhante. A caracterização dos compostos e os símbolos usados para a sua identificação encontram-se em Leite (1988).

Foram realizados 22 ensaios, nos anos de 1985 a 1991, nas seguintes localidades: Viçosa (1986), Coimbra (1986, 1987, 1988, 1990 e 1991), Leopoldina (1986), Ponte Nova (1986 e 1988), Patos de Minas (1986), Sete Lagoas (1986), Marreco (1988), Teixeiras (1988 e1989), Visconde do Rio Branco (1988, 1990 e 1991) e Nova Viçosa (1988), municípios de Minas Gerais, e Campos (1989), Avelar (1989 e

Tabela 1. Identificação dos tratamentos usados nos 22 ensaios.

\begin{tabular}{|c|c|c|c|}
\hline Tratamento & Identificação & Descrição & Programa de origem ${ }^{(1)}$ \\
\hline 1 & L-SRR-D 84-4 x L-SRR-F 84-4 & Híbrido de progênie $S_{1}$ & A \\
\hline 2 & L-SRR-D 85-2 x L-SRR-F 85-2 & Híbrido de progênie $S_{3}$ & B \\
\hline 3 & L-SRR-D 85-3 x L-SRR-F 85-3 & Híbrido de progênie $S_{3}$ & B \\
\hline 4 & L-SRR-D 84-5 x L-SRR-F 84-5 & Híbrido de progênie $S_{1}$ & A \\
\hline 5 & L-SRR-D 85-1 x L-SRR-F 85-1 & Híbrido de progênie $S_{2}$ & A \\
\hline 6 & L-SRR-D 86-2 x L-SRR-F 86-2 & Híbrido de progênie $S_{2}$ & A \\
\hline 7 & L-SRR-D 86-1 x L-SRR-F 86-1 & Híbrido de progênie $S_{4}$ & B \\
\hline 8 & L-SRR-D 86-11 x L-SRR-F 86-11 & Híbrido de progênie $S_{2}$ & B \\
\hline 9 & L-SRR-D 86-9 x L-SRR-F 86-9 & Híbrido de progênie $S_{2}$ & B \\
\hline 10 & L-SRR-D 86-10 x L-SRR-F 86-10 & Híbrido de progênie $S_{3}$ & B \\
\hline 11 & L-SRR-D 86-29 x L-SRR-F 86-29 & Híbrido de progênie $S_{2}$ & B \\
\hline 12 & L-SRR-D 86-8 x L-SRR-F 86-8 & Híbrido de progênie $S_{2}$ & B \\
\hline 13 & L-SRR-D 86-15 x L-SRR-F 86-15 & Híbrido de progênie $S_{2}$ & B \\
\hline 14 & L-SRR-D 86-19 x L-SRR-F 86-19 & Híbrido de progênie $S_{2}$ & B \\
\hline 15 & L-SRR-D 86-31 x L-SRR-F 86-31 & Híbrido de progênie $S_{1}$ & B \\
\hline 16 & L-SRR-D 86-28 x L-SRR-F 86-28 & Híbrido de progênie $S_{2}$ & B \\
\hline 17 & L-SRR-D 86-27 x L-SRR-F 86-27 & Híbrido de progênie $S_{2}$ & B \\
\hline 18 & L-SRR-D 86-22 x L-SRR-F 86-22 & Híbrido de progênie $S_{2}$ & B \\
\hline 19 & L-SRR-D 86-21 x L-SRR-F 86-21 & Híbrido de progênie $S_{2}$ & B \\
\hline 20 & L-SRR-D 86-16 x L-SRR-F 86-16 & Híbrido de progênie $S_{2}$ & B \\
\hline 21 & L-SRR-D 86-23 x L-SRR-F 86-23 & Híbrido de progênie $S_{2}$ & B \\
\hline 22 & L-SRR-D 84-6 x L-SRR-F 84-6 & Híbrido de progênie $S_{1}$ & A \\
\hline 23 & L-SRR-D 86-26 x L-SRR-F 86-26 & Híbrido de progênie $S_{2}$ & B \\
\hline 24 & $\mathrm{CD}_{\mathrm{S}_{1 \mathrm{n}} \mathrm{S}_{2}} \overline{\mathrm{S}_{1} \mathrm{~S}_{2}} \times \mathrm{CF} \overline{\mathrm{S}_{1 \mathrm{n}} \mathrm{S}_{2}} \overline{\mathrm{S}_{1} \mathrm{~S}_{2}}$ & Testemunha & $\mathrm{C}$ \\
\hline 25 & $\mathrm{CDE} \overline{\mathrm{S}_{1 \mathrm{n}} \mathrm{S}_{2}} \overline{\mathrm{S}_{1 \mathrm{n}} * \mathrm{~S}_{2}} \times \mathrm{CFE} \overline{\mathrm{S}_{1 \mathrm{n}} \mathrm{S}_{2}} \overline{\mathrm{S}_{1 \mathrm{n}} * \mathrm{~S}_{2}}$ & Testemunha & $\mathrm{C}$ \\
\hline 26 & $\mathrm{CDE} \overline{\mathrm{S}_{1 \mathrm{n}} \mathrm{S}_{2 \mathrm{n}} \mathrm{S}_{3}} *$ x CFE $\overline{\mathrm{S}_{1 \mathrm{n}} \mathrm{S}_{2 \mathrm{n}} \mathrm{S}_{3}} *$ & Testemunha & $\mathrm{C}$ \\
\hline 27 & $\mathrm{CD} \overline{\mathrm{S}_{1 \mathrm{n}} \mathrm{S}_{2 \mathrm{n}} \mathrm{S}_{3}} \times \mathrm{CF} \overline{\mathrm{S}_{1 \mathrm{n}} \mathrm{S}_{2 \mathrm{n}} \mathrm{S}_{3}}$ & Testemunha & $\mathrm{C}$ \\
\hline 28 & P-SRR-D 86-4 x P-SRR-F 86-4 & Testemunha & $\mathrm{C}$ \\
\hline 29 & P-SRR-D 86-3 x P-SRR-F 86-3 & Testemunha & $\mathrm{C}$ \\
\hline
\end{tabular}

${ }^{(1)} \mathrm{A}$ : programa com compostos produzidos por $\mathrm{SRR}$, pela recombinação de progênies $\mathrm{S}_{4}$ não selecionadas; B: programa com compostos produzidos por $\mathrm{SRR}$, pela recombinação de progênies $\mathrm{S}_{2}$ selecionadas; C: programa de SRR para melhoramento do composto e posterior obtenção do híbrido. 
1990) e Itaguaí (1990), municípios do Rio de Janeiro Considerou-se que cada ensaio representava um ambiente, uma vez que foram conduzidos em condições diferentes de solo, clima, densidade e época de plantio. Foi efetuada uma análise de variância para cada ensaio. Posteriormente, foi feita a análise conjunta, incluindo todos os ensaios, uma vez que a relação entre o menor quadrado médio de resíduo não diferiu em mais de sete vezes do maior, havendo homogeneidade das variâncias residuais (Pimentel-Gomes, 1987). Os parâmetros de adaptabilidade foram estimados usando-se os métodos de Eberhart \& Russell (1966) e Carneiro (1998).

\section{Método de Eberhart \& Russell (1966)}

No método proposto por Eberhart \& Russell (1966), faz-se uma análise de regressão linear com relação a cada população, em razão de um índice de ambiente, definido como a diferença entre a média dos genótipos avaliados e a média de todos os locais. Obtém-se, assim, para cada genótipo, o coefíciente de regressão $\left(\hat{\beta}_{\mathrm{li}}\right)$ e o componente de variância associado aos desvios de regressão $\left(\hat{\sigma}_{\mathrm{di}}\right)$. Quando $\beta_{1 \mathrm{i}}<1,0$, o genótipo responde pouco à melhoria do ambiente, e é considerado de comportamento relativamente melhor em ambientes desfavoráveis. Quando $\beta_{\mathrm{li}}>1,0$, a população responde de forma acentuada a esta melhoria. Neste caso, seu comportamento é relativamente superior nos melhores ambientes. Se $\beta_{\mathrm{li}}=1,0$, a população tende a ter bom desempenho em qualquer ambiente, $\mathrm{e}$ é considerada com adaptabilidade geral ou ampla. $\mathrm{O}$ desvio de regressão mede a estabilidade de comportamento da cultivar. Se $\hat{\sigma}_{\mathrm{di}}=0$, a cultivar exibe alta estabilidade, e seu comportamento, quanto ao rendimento, é previsível nos distintos ambientes. Para uma adequada caracterização dos padrões relativos de adaptabilidade, deve-se considerar a média da população

\section{Método de Carneiro (1998)}

A estimativa do parâmetro de estabilidade em plantas foi definida por Lin \& Binns (1988) como o quadrado médio da distância entre a média da cultivar e a resposta média máxima, em todos os locais. Carneiro (1998) propôs a decomposição do estimador do $\mathrm{P}_{\mathrm{i}}$ (parâmetro de desempenho genotípico) em ambientes favoráveis e desfavoráveis. A classificação destes ambientes foi feita com base nos índices ambientais. Foram realizadas estimativas de médias de produtividade de grãos, do $\mathrm{P}_{\mathrm{i}}$ (parâmetro de desempenho genotípico), do $\mathrm{P}_{\text {if }}$ (parâmetro de medida de adaptabilidade e estabilidade em ambientes favoráveis) e do $P_{\text {id }}$ (parâmetro de medida de adaptabilidade e estabilidade em ambientes desfavoráveis), das populações de milho avaliadas nos 22 ensaios, referentes às safras agrícolas de 1986 a 1992.

\section{Resultados e Discussão}

\section{Método de Eberhart \& Russell (1966)}

Os resultados da análise de variância conjunta, com a decomposição da soma de quadrados de ambientes "dentro" de tratamentos, referente à produção de grãos de 29 populações de milho, comuns a 22 ensaios, encontram-se na Tabela 2. A significância do teste de ambiente linear indica que existem variações significativas nos ambientes para proporcionar alterações nas médias dos tratamentos. O teste de tratamento $\mathrm{x}$ ambiente linear foi significativo, indicando diferenças genéticas entre os tratamentos quanto às suas respostas às variações ambientais. Logo, existem diferenças entre os coeficientes de regressão do grupo de populações avaliadas.

Ambiente favorável é aquele que possibilita média de produção das cultivares maior que a média geral de todos os ensaios, apresentando, portanto, índice positivo. Foram caracterizados como favoráveis: Viçosa (1986), Leopoldina (1986), Sete Lagoas (1986), Patos de Minas (1986), Coimbra (1987, 1988, 1990, 1991), Marreco (1988), Nova Viçosa (1988), Ponte Nova (1988) e Visconde do Rio Branco (1990). Os ambientes Coimbra (1986), Ponte Nova (1986), Teixeiras (1988, 1989), Visconde do Rio Branco (1988, 1991), Campos (1989), Avelar (1989 e 1990) e Itaguaí (1990) foram considerados como desfavoráveis, por propiciarem índices ambientais negativos.

A caracterização dos 29 tratamentos quanto aos padrões relativos de adaptabilidade e estabilidade, segundo o método proposto por Eberhart \& Russell (1966), encontra-se na Tabela 3. Dos 23 híbridos de famílias endogâmicas, 74\% apresentaram ampla adaptabilidade (tratamentos 1, 3, 5, 6, 7, 8, 10, 11, $13,14,15,16,17,19,20,21$ e 22 ), $17 \%$ foram mais responsivos à melhoria do ambiente (tratamentos 2 , 4,12 e 18 ), e $9 \%$ foram mais adaptados a ambientes desfavoráveis (tratamentos 9 e 23). Quanto à estabilidade, aproximadamente $74 \%$ desses híbridos apresentaram estabilidade alta (tratamentos 2, 4, 7, 8, 9, $10,11,13,14,15,17,18,19,20,21,22$ e 23 ) e $26 \%$ apresentaram estabilidade baixa (tratamentos 1, 3, $5,6,12$ e 16). Na análise de cada parâmetro, foi possível observar que a maioria dos híbridos apresenta- 
ram bom desempenho em qualquer ambiente e que a maioria apresentou estabilidade alta. Podemos observar, ainda, que aproximadamente 52\% dos 23 híbridos crípticos apresentaram ampla adaptabilidade e estabilidade alta (tratamentos 7, 8, 10, 11, 13, 14,

Tabela 2. Resumo da análise de variância conjunta da produção de grãos (kg/ha) de 23 híbridos de famílias endogâmicas e seis híbridos de compostos, com a decomposição da soma de quadrados de ambientes/tratamentos, segundo Eberhart \& Russell (1966).

\begin{tabular}{|c|c|c|}
\hline Fonte de variação & G.L. & Quadrado médio \\
\hline Blocos/Ambientes & 22 & - \\
\hline Tratamentos & 28 & $5.833 .190,0^{*}$ \\
\hline Ambientes & 21 & $162.883 .190,9^{*}$ \\
\hline Trat. x Amb. & 588 & $1.197 .453,0^{*}$ \\
\hline Amb./Trat. & 609 & $6.772 .823,3$ \\
\hline Ambientes (linear) & 1 & $3.420 .547 .009,5^{*}$ \\
\hline Trat. x Amb. linear & 28 & $1.905 .841,4^{*}$ \\
\hline Desvios combinados & 580 & $1.121 .963,4^{*}$ \\
\hline 1 & 20 & $1.430 .231,5^{*}$ \\
\hline 2 & 20 & $1.006 .006,3^{\mathrm{ns}}$ \\
\hline 3 & 20 & $1.569 .865,3^{\mathrm{ns}}$ \\
\hline 4 & 20 & $878.401,5^{\mathrm{ns}}$ \\
\hline 5 & 20 & $1.423 .181,2^{\mathrm{ns}}$ \\
\hline 6 & 20 & $1.343 .215,9^{\mathrm{ns}}$ \\
\hline 7 & 20 & $772.311,3^{\mathrm{ns}}$ \\
\hline 8 & 20 & $1.218 .332,3^{\mathrm{ns}}$ \\
\hline 9 & 20 & $637.438,3^{\mathrm{ns}}$ \\
\hline 10 & 20 & $1.276 .030,3^{\mathrm{ns}}$ \\
\hline 11 & 20 & $852.155,9^{\mathrm{ns}}$ \\
\hline 12 & 20 & $1.424 .982,6^{*}$ \\
\hline 13 & 20 & $1.234 .950,2^{\mathrm{ns}}$ \\
\hline 14 & 20 & $1.125 .649,9^{\mathrm{ns}}$ \\
\hline 15 & 20 & $1.083 .486,6^{\mathrm{ns}}$ \\
\hline 16 & 20 & $1.678 .872,8^{*}$ \\
\hline 17 & 20 & $1.003 .133,3^{\mathrm{ns}}$ \\
\hline 18 & 20 & $1.066 .573,6^{\mathrm{ns}}$ \\
\hline 19 & 20 & $958.601,7^{\mathrm{ns}}$ \\
\hline 20 & 20 & $864.239,9^{\mathrm{ns}}$ \\
\hline 21 & 20 & $1.297 .700,7^{\mathrm{ns}}$ \\
\hline 22 & 20 & $807.071,9^{\mathrm{ns}}$ \\
\hline 23 & 20 & $1.018 .214,7^{\mathrm{ns}}$ \\
\hline 24 & 20 & $1.524 .047,2^{*}$ \\
\hline 25 & 20 & $1.019 .549,0^{\mathrm{ns}}$ \\
\hline 26 & 20 & $880.040,0^{\mathrm{ns}}$ \\
\hline 27 & 20 & $860.027,8^{\mathrm{ns}}$ \\
\hline 28 & 20 & $876.726,2^{\mathrm{ns}}$ \\
\hline 29 & 20 & $1.405 .902,2^{*}$ \\
\hline Resíduo & 616 & $844.632,9$ \\
\hline
\end{tabular}

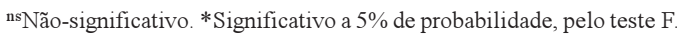

$15,17,19,20,21$ e 22 ) e $22 \%$ apresentaram ampla adaptabilidade e estabilidade baixa (tratamentos 1 , $3,5,6$, e 16). Treze por cento foram mais responsivos à melhoria do ambiente e apresentaram estabilidade alta (tratamentos 2, 4 e 18), e apenas o tratamento 12 foi mais responsivo à melhoria do ambiente, e de estabilidade baixa, representando aproximadamente 4\% dos 23 híbridos crípticos. Nove por cento apresentaram adaptabilidade a ambientes desfavoráveis, e estabilidade alta (tratamentos 9 e 23) e não foram encontrados híbridos adaptados a ambientes desfavoráveis e de baixa estabilidade. Analisando simultaneamente a adaptabilidade e a estabilidade dos híbridos de famílias endogâmicas, foi possível observar a predominância de híbridos de padrões de ampla adaptabilidade e estabilidade alta. Em relação aos

Tabela 3. Estimativas dos parâmetros de adaptabilidade e estabilidade obtidos pelo método de Eberhart \& Russell (1966)

\begin{tabular}{|c|c|c|c|c|}
\hline Tratamento & $\begin{array}{l}\text { Média } \\
\text { (kg/ha) }\end{array}$ & $\begin{array}{l}\text { Coeficiente de } \\
\text { regressão }\left(\hat{\beta}_{1 \mathrm{i}}\right)\end{array}$ & $\begin{array}{c}\text { Desvio de } \\
\text { regressão }\left(\hat{\sigma}_{\mathrm{di}}^{2}\right)\end{array}$ & $\mathrm{R}^{2}$ \\
\hline 1 & $4.588,0$ & $0,92 \mathrm{~ns}$ & $292.799,2^{*}$ & 0,778 \\
\hline 2 & $5.640,7$ & $1,18^{*}$ & $80.686,6 \mathrm{~ns}$ & 0,890 \\
\hline 3 & $5.645,5$ & $0,87 \mathrm{~ns}$ & $362.616,1^{*}$ & 0,742 \\
\hline 4 & $5.701,6$ & $1,26^{*}$ & $16.884,2 \mathrm{~ns}$ & 0,914 \\
\hline 5 & $5.359,1$ & $1,00 \mathrm{~ns}$ & $289.274,1 *$ & 0,806 \\
\hline 6 & $5.595,0$ & $1,07 \mathrm{~ns}$ & $249.291,4^{*}$ & 0,836 \\
\hline 7 & $5.384,6$ & $0,98 \mathrm{~ns}$ & $-36.160,8 \mathrm{~ns}$ & 0,880 \\
\hline 8 & $5.547,2$ & $0,84 \mathrm{~ns}$ & $186.849,6 \mathrm{~ns}$ & 0,777 \\
\hline 9 & $5.077,9$ & $0,66^{*}$ & $-103.597,3 \mathrm{~ns}$ & 0,804 \\
\hline 10 & $5.419,0$ & $1,06 \mathrm{~ns}$ & $215.698,6 \mathrm{~ns}$ & 0,839 \\
\hline 11 & $5.005,0$ & $0,94 \mathrm{~ns}$ & $3.761,5 \mathrm{~ns}$ & 0,860 \\
\hline 12 & $5.644,2$ & $1,22 *$ & $290.174,8 *$ & 0,862 \\
\hline 13 & $5.500,6$ & $0,97 \mathrm{~ns}$ & $195.158,6 \mathrm{~ns}$ & 0,818 \\
\hline 14 & $5.618,4$ & $1,11 \mathrm{~ns}$ & $140.508,4 \mathrm{~ns}$ & 0,866 \\
\hline 15 & $4.978,0$ & $0,90 \mathrm{~ns}$ & $119.426,8 \mathrm{~ns}$ & 0,817 \\
\hline 16 & $4.318,2$ & $0,86 \mathrm{~ns}$ & $417.119,9 *$ & 0,726 \\
\hline 17 & $5.434,9$ & $0,93 \mathrm{~ns}$ & $79.250,1 \mathrm{~ns}$ & 0,836 \\
\hline 18 & $5.780,0$ & $1,18^{*}$ & $110.970,3 \mathrm{~ns}$ & 0,886 \\
\hline 19 & $5.492,3$ & $0,95 \mathrm{~ns}$ & $56.984,3 \mathrm{~ns}$ & 0,849 \\
\hline 20 & $5.212,7$ & $1,04 \mathrm{~ns}$ & $9.803,4 \mathrm{~ns}$ & 0,881 \\
\hline 21 & $5.165,9$ & $1,00 \mathrm{~ns}$ & $226.533,8 \mathrm{~ns}$ & 0,820 \\
\hline 22 & $5.346,6$ & $1,06 \mathrm{~ns}$ & $-18.780,5 \mathrm{~ns}$ & 0,892 \\
\hline 23 & $4.627,4$ & $0,83^{*}$ & $86.790,8 \mathrm{~ns}$ & 0,800 \\
\hline 24 & $5.411,5$ & $1,01 \mathrm{~ns}$ & $339.707,1^{*}$ & 0,799 \\
\hline 25 & $5.360,6$ & $1,04 \mathrm{~ns}$ & $87.458,0 \mathrm{~ns}$ & 0,862 \\
\hline 26 & $5.681,8$ & $1,07 \mathrm{~ns}$ & $17.703,5 \mathrm{~ns}$ & 0,884 \\
\hline 27 & $4.996,2$ & $1,02 \mathrm{~ns}$ & $7.697,4 \mathrm{~ns}$ & 0,878 \\
\hline 28 & $5.702,9$ & $0,96 \mathrm{~ns}$ & $16.046,6 \mathrm{~ns}$ & 0,861 \\
\hline 29 & $5.209,0$ & $0,94 \mathrm{~ns}$ & $280.634,6^{*}$ & 0,787 \\
\hline
\end{tabular}

nsNão-significativo. * Significativo a $5 \%$ de probabilidade, pelo teste $\mathrm{F}$. 
híbridos de compostos, 100\% apresentaram ampla adaptabilidade, e $80 \%$, alta estabilidade. Apenas o tratamento 29 apresenta ampla adaptabilidade, associada a baixa estabilidade. A predominância da ampla adaptabilidade e estabilidade alta destes materiais é atribuída ao fato de populações heterogêneas com grande número de genótipos possuírem maior capacidade adaptativa e maior estabilidade Paterniani (1965).

$\mathrm{O}$ tratamento 12 foi o mais responsivo à melhoria ambiental e apresentou baixa estabilidade, sendo o quarto híbrido mais produtivo. Este e os tratamentos 5 e 6, também com boa produção média, apresentaram coeficiente de determinação $\left(\mathrm{R}^{2}\right)$ acima de $80 \%$, o que indica que o modelo de regressão linear pode ser considerado satisfatório para descrever seus comportamentos.

\section{Método de Carneiro (1998)}

As estimativas de médias de produtividade de grãos, do $\mathrm{P}_{\mathrm{i}}$, do $\mathrm{P}_{\text {if }}$ e do $\mathrm{P}_{\mathrm{id}}$ das populações de milho encontram-se na Tabela 4. Os quinze híbridos de famílias endogâmicas com bom desempenho em qualquer ambiente, e maior estabilidade, com base no parâmetro $\mathrm{P}_{\mathrm{i}}$ desenvolvido por Lin \& Binns (1988), em ordem crescente de $\mathrm{P}_{\mathrm{i}}$, correspondem aos tratamentos 18, 14, 2, 3, 4, 6, 12, 19, 8, 13, 7, 17, 5, 22 e 10. Considerando as estimativas de $\mathrm{P}_{\mathrm{i}}$ desses híbridos de famílias endogâmicas, relativos às duas classes de ambientes, os cinco tratamentos mais responsivos à melhoria do ambiente e com alta estabilidade, em ordem crescente de $\mathrm{P}_{\text {if }}$, são: $18,12,4,2$ e 14. Os cinco híbridos de famílias endogâmicas com bom desempenho em ambientes desfavoráveis, e mais estáveis, em ordem crescente de $\mathrm{P}_{\text {id }}$, são: tratamentos $8,3,17,6$ e 13 .

Onze dos quinze híbridos de famílias endogâmicas mais estáveis segundo a método de Carneiro (1998) também são considerados de estabilidade alta segundo o método de Eberhart \& Russell (1966) (tratamentos $18,14,2,4,19,8,13,7,17,22$ e 10). Dos cinco híbridos discriminados pelo método de Carneiro (1998) como os mais adaptados a ambientes favoráveis, quatro foram assim caracterizados pelo método de Eberhart \& Russell (1966) (tratamentos $18,12,4$ e 2 ). Apenas o tratamento 14 , que apresen- tou ampla adaptabilidade pelo método de Eberhart \& Russell (1966), foi classificado pelo método de Carneiro (1998) como mais adaptado a ambientes favoráveis. Pelo método de Eberhart \& Russell (1966), só foram encontrados dois híbridos de famílias endogâmicas mais adaptados a ambientes desfavoráveis (tratamentos 9 e 23). O tratamento 23 é estatisticamente inferior aos tratamentos 18 e 26 (Tabela 5). Se for necessário indicar, além do tratamento 9 , outros híbridos de famílias endogâmicas adaptados a ambientes desfavoráveis, é possível recorrer ao método de Carneiro (1998), e indicar os tratamentos $8,3,17,6$ e 13 , os quais estão entre os 17 híbri-

Tabela 4. Estimativas da média de produtividade de grãos ( $\mathrm{kg} / \mathrm{ha})$, do parâmetro de desempenho genotípico em ambientes favoráveis e desfavoráveis $\left(\mathrm{P}_{\mathrm{i}}\right)$, do parâmetro de medida de adaptabilidade e estabilidade de ambientes favoráveis $\left(\mathrm{P}_{\text {if }}\right)$ e do parâmetro de medida de adaptabilidade e estabilidade em ambientes desfavoráveis $\left(\mathrm{P}_{\mathrm{id}}\right)$ pelo método de Lin e Binns com decomposição do parâmetro $\mathrm{P}_{\mathrm{i}}$, em relação a híbridos de famílias endogâmicas e de compostos.

\begin{tabular}{rrrrr}
\hline Tratamento & Média & \multicolumn{1}{c}{$\mathrm{P}_{\mathrm{i}}$} & \multicolumn{1}{c}{$\mathrm{P}_{\mathrm{if}}$} & \multicolumn{1}{c}{$\mathrm{P}_{\mathrm{id}}$} \\
\hline 18 & $5.780,0$ & $971.679,9$ & $908.938,1$ & $1.046 .970,1$ \\
14 & $5.618,4$ & $1.096 .887,6$ & $1.012 .787,9$ & $1.197 .807,3$ \\
2 & $5.640,7$ & $1.125 .211,7$ & $997.598,9$ & $1.278 .347,2$ \\
3 & $5.645,5$ & $1.148 .403,5$ & $1.461 .423,6$ & $772.779,4$ \\
4 & $5.701,6$ & $1.209 .798,1$ & $996.349,8$ & $1.465 .936,2$ \\
28 & $5.702,9$ & $1.214 .609,0$ & $1.499 .592,1$ & $872.629,2$ \\
6 & $5.595,0$ & $1.224 .383,6$ & $1.463 .011,9$ & $938.029,6$ \\
26 & $5.681,8$ & $1.229 .220,5$ & $1.288 .767,7$ & $1.157 .763,9$ \\
12 & $5.644,2$ & $1.299 .549,2$ & $920.292,2$ & $1.754 .657,5$ \\
19 & $5.492,3$ & $1.438 .497,2$ & $1.508 .639,5$ & $1.354 .326,5$ \\
8 & $5.547,2$ & $1.476 .907,5$ & $2.157 .521,3$ & $660.171,0$ \\
13 & $5.500,6$ & $1.497 .641,4$ & $1.836 .831,9$ & $1.090 .613,0$ \\
7 & $5.384,6$ & $1.578 .814,8$ & $1.729 .911,9$ & $1.397 .498,3$ \\
25 & $5.360,6$ & $1.585 .916,3$ & $1.752 .186,6$ & $1.386 .392,0$ \\
17 & $5.434,9$ & $1.598 .382,8$ & $2.161 .509,9$ & $922.630,4$ \\
5 & $5.359,1$ & $1.670 .136,9$ & $2.032 .523,6$ & $1.235 .272,9$ \\
22 & $5.346,6$ & $1.678 .595,9$ & $1.747 .566,9$ & $1.595 .830,6$ \\
10 & $5.419,0$ & $1.752 .501,5$ & $1.726 .564,2$ & $1.783 .626,3$ \\
24 & $5.411,5$ & $1.825 .974,8$ & $2.312 .461,4$ & $1.242 .191,0$ \\
20 & $5.212,7$ & $1.970 .173,2$ & $2.003 .811,7$ & $1.833 .807,0$ \\
21 & $5.165,9$ & $2.227 .840,8$ & $2.627 .592,5$ & $1.748 .138,8$ \\
29 & $5.209,0$ & $2.278 .168,5$ & $2.771 .083,9$ & $1.686 .670,0$ \\
11 & $5.005,0$ & $2.324 .026,0$ & $2.812 .335,9$ & $1.738 .054,2$ \\
9 & $5.077,9$ & $2.359 .871,7$ & $3.460 .694,0$ & $1.038 .884,9$ \\
27 & $4.996,2$ & $2.391 .867,4$ & $2.655 .819,7$ & $2.075 .124,8$ \\
15 & $4.978,0$ & $2.443 .508,7$ & $2.847 .330,1$ & $1.958 .923,0$ \\
23 & $4.627,4$ & $3.005 .951,1$ & $3.833 .068,7$ & $2.013 .409,9$ \\
1 & $4.588,0$ & $3.418 .734,1$ & $4.077 .085,5$ & $2.628 .712,4$ \\
16 & $4.318,2$ & $4.255 .370,2$ & $4.757 .818,0$ & $3.652 .432,8$ \\
\hline & & & & \\
& & & &
\end{tabular}


dos que apresentam ampla adaptabilidade pelo método de Eberhart \& Russell (1966).

O uso dos dois métodos para caracterização dos híbridos foi realizado principalmente para se ter maior segurança na seleção dos pares de famílias endogâmicas que darão continuidade ao programa de melhoramento. A complementação de informações e a confirmação de grande parte dos resultados dos dois métodos aumentam a segurança no processo de

Tabela 5. Comparação entre as médias de produção de grãos (kg/ha) de 23 híbridos de famílias endogâmicas e 6 híbridos de compostos ${ }^{(1)}$.

\begin{tabular}{|c|c|}
\hline Tratamento & Média \\
\hline 18 & $5.780,0 \mathrm{a}$ \\
\hline 28 & $5.618,4 \mathrm{a}$ \\
\hline 4 & $5.640,7 \mathrm{a}$ \\
\hline 26 & $5.645,5 \mathrm{a}$ \\
\hline 3 & $5.701,6 a b$ \\
\hline 12 & $5.702,9 \mathrm{ab}$ \\
\hline 2 & $5.595,0 \mathrm{ab}$ \\
\hline 14 & $5.681,8 \mathrm{abc}$ \\
\hline 6 & $5.644,2 \mathrm{abc}$ \\
\hline 8 & $5.492,3 \mathrm{abc}$ \\
\hline 13 & $5.547,2 \mathrm{abc}$ \\
\hline 19 & $5.500,6 \mathrm{abc}$ \\
\hline 17 & $5.384,6 a b c$ \\
\hline 10 & $5.360,6 \mathrm{abc}$ \\
\hline 24 & $5.434,9 \mathrm{abc}$ \\
\hline 7 & $5.359,1 \mathrm{abc}$ \\
\hline 25 & $5.346,6 \mathrm{abcd}$ \\
\hline 5 & $5.419,0 \mathrm{abcd}$ \\
\hline 22 & $5.411,5 \mathrm{abcd}$ \\
\hline 20 & $5.212,7 \mathrm{abcd}$ \\
\hline 29 & $5.165,9 \mathrm{abcd}$ \\
\hline 21 & $5.209,0 \mathrm{abcd}$ \\
\hline 9 & $5.005,0 \mathrm{abcd}$ \\
\hline 11 & $5.077,9 \mathrm{abcd}$ \\
\hline 27 & $4.996,2 \mathrm{abcd}$ \\
\hline 15 & $4.978,0 \mathrm{abcd}$ \\
\hline 23 & $4.627,4 \mathrm{bcd}$ \\
\hline 1 & $4.588,0 \mathrm{~cd}$ \\
\hline 16 & $4.318,2 \mathrm{~d}$ \\
\hline
\end{tabular}

(1) Médias seguidas de mesma letra não diferem entre si a 5\% de probabilidade, pelo teste de Tukey seleção, em virtude da obtenção de resultados similares entre métodos de princípios diferentes.

Não foram observadas diferenças estatísticas significativas entre as médias de 20 híbridos de famílias endogâmicas dos 23 testados e as médias dos seis compostos utilizados como testemunhas, o que indica o bom desempenho desses materiais. Os híbridos de famílias endogâmicas referentes aos tratamentos 1 e 23 apresentaram médias que não diferiram estatisticamente de quatro das seis testemunhas (tratamentos 24, 25, 27 e 29), e o híbrido referente ao tratamento 16 apresentou média que não diferiu estatisticamente de dois híbridos de compostos (tratamentos 27 e 29). Estes resultados são decorrentes do fato de esses materiais já terem passado por seleção para capacidade produtiva (Lopes, 1999).

A caracterização dos híbridos de famílias endogâmicas quanto a seus padrões relativos de adaptabilidade e estabilidade após o processo de seleção para capacidade produtiva é de grande importância para a seleção dos de famílias endogâmicas a serem usados para dar continuidade ao programa de melhoramento. Com base nos padrões de adaptabilidade e estabilidade dos híbridos crípticos, podemos observar que entre os 15 mais produtivos (tratamentos $18,4,3,12,2,14,6,8,13,19,17,10,7,5$ e 22$)$ existem materiais com comportamentos diversos (de estabilidade alta associada a ampla adaptabilidade, ou a responsivos à melhoria do ambiente ou adaptados a ambientes desfavoráveis) que seriam interessantes para um programa de melhoramento (Tabelas 3 e 4). Visando desenvolver materiais para um amplo conjunto de ambientes, espera-se que a continuidade do processo, com a seleção dos pares de famílias endogâmicas correspondentes a estes híbridos, permitirá obter híbridos simples, duplos e triplos com elevada capacidade produtiva, com estabilidade alta e com adaptação a diversos ambientes.

Desses 15 híbridos, segundo o método de Eberhart \& Russell (1966), 8 híbridos (53,3\%) apresentaram bom desempenho em qualquer ambiente e alta estabilidade, devendo-se ressaltar que neste grupo estão, respectivamente, 50, 55,5, 33,3 e 100\% dos de famílias $\mathrm{S}_{1}, \mathrm{~S}_{2}, \mathrm{~S}_{3}$ e $\mathrm{S}_{4}$ avaliados. Os responsivos à melhoria ambiental e de alta estabilidade representam 20\% (3 híbridos), dos 15 mais produtivos. Nesta classe estão 50, 15,6 e 33,3\% dos híbridos de famí- 
lias $\mathrm{S}_{1}, \mathrm{~S}_{2}$ e $\mathrm{S}_{3}$ avaliados, respectivamente. Os híbridos que apresentaram bom desempenho em qualquer ambiente e baixa estabilidade correspondem a 3 híbridos (20\% dos 15 híbridos). Nesta categoria estão 22,2\% dos de famílias $\mathrm{S}_{2}$ avaliados, e 33,3\% dos de famílias $S_{3}$. A menor proporção $(6,7 \%)$ corresponde ao de famílias $\mathrm{S}_{2}$, que é responsivo à melhoria ambiental e apresenta baixa estabilidade.

Três desses híbridos selecionados são derivados do programa com compostos produzidos pela recombinação de progênies $\mathrm{S}_{4}$ não selecionadas, dois (66,7\%) são híbridos de ampla adaptabilidade e baixa estabilidade, e um (33,3\%) é híbrido responsivo à melhoria ambiental e de alta estabilidade. Doze dos 15 híbridos são obtidos do programa com compostos produzidos pela recombinação de progênies $\mathrm{S}_{2}$ selecionadas, 66,7, 16,7, 8,3 e 8,3\% correspondem, respectivamente, aos que apresentam bom desempenho em qualquer ambiente e alta estabilidade, aos mais responsivos à melhoria ambiental e com alta estabilidade, ao que apresenta bom desempenho em qualquer ambiente e baixa estabilidade e ao de bom desempenho em qualquer ambiente e de baixa estabilidade

As famílias "dent" e "flint" 84-5, 85-1 e 86-2, as quais são derivadas do programa com compostos produzidos pela recombinação de progênies $\mathrm{S}_{4}$ não selecionadas, deram origem a 75\% (três em quatro) destes híbridos. As demais famílias citadas foram obtidas do programa envolvendo compostos produzidos pela recombinação de progênies $\mathrm{S}_{2}$ selecionadas, e os híbridos deste programa representam aproximadamente $63,1 \%$ dos que foram avaliados (doze em dezenove). Dos híbridos $\mathrm{S}_{1} \times \mathrm{S}_{1}$ incluídos na análise conjunta, $50 \%$ (dois em quatro) encontram-se entre os quinze híbridos relatados acima. Dentre estes, os valores correspondentes aos de progênies $S_{2}, S_{3}$ e $S_{4}$ são, respectivamente, $60 \%$ (nove em quinze), $100 \%$ (três em três) e 100\% (quatro em quatro). Portanto, a avaliação de híbridos $S_{1} \times S_{1}$ pode permitir identificar pares de famílias nas quais deve ser possível, após as autofecundações, obter linhagens capazes de gerar híbridos superiores.

A partir dos resultados obtidos nesse trabalho, será realizada a próxima etapa do programa de melhoramento de milho, a extração de linhagens a partir dos 15 pares de famílias endogâmicas selecionadas que deram origem aos tratamentos: 4 e 22 de progênies $\mathrm{S}_{1} ; 5,6,8,12,13,14,17,18$ e 19 de progênies $\mathrm{S}_{2} ; 2$, 3 e 10 de progênies $S_{3}$; e 7 de progênies $S_{4}$.

\section{Conclusões}

1. Entre os híbridos crípticos selecionados predomina o padrão de adaptabilidade geral e alta estabilidade.

2. Recomenda-se para dar continuidade ao programa de melhoramento quinze pares de famílias endogâmicas, sendo 2, 9, 3 e 1 pares de famílias $S_{1}$, $\mathrm{S}_{2}, \mathrm{~S}_{3}$ e $\mathrm{S}_{4}$, respectivamente.

\section{Agradecimentos}

Ao Dr. José Carlos Silva, pela condução do programa de melhoramento que deu origem a este trabalho.

\section{Referências}

ALLARD, R. W. Relationship between genetic diversity and consistence of performance in different environments. Crop Science, Madison, v. 11, n. 2, p. 127-133, 1971.

ALLARD, R. W.; BRADSHAW, A. D. Implications of genotype-environmental interactions in applied plant breeding. Crop Science, Madison, v. 4, n. 5, p. 503-508, 1964.

BORÉM, A. Melhoramento de plantas. 2. ed. Viçosa : UFV, 1999. $453 \mathrm{p}$

CARNEIRO, P. C. S. Novas metodologias de análise da adaptabilidade e estabilidade de comportamento. Viçosa : UFV, 1998. 168 p. Tese de Doutorado.

COSTA, S. N. Interação cultivares de milho (Zea mays $L$.) $x$ anos $x$ localidades nos Estados de Piauí e Maranhão - Brasil. Piracicaba : ESALQ, 1976. 82 p. Dissertação de Mestrado.

EBERHART, S. A.; RUSSELL, W. A. Stability parameters for comparing varieties. Crop Science, Madison, v. 6, n. 1, p. 36-40, 1966.

EBERHART, S. A.; RUSSELL, W. A. Yield and stability for a line diallel of single-cross and double-cross maize hybrids. Crop Science, Madison, v. 9, n. 3, p. 357-361, 1969. 
EBERHART, S. A.; RUSSELL, W. A.; PENNY, L. H. Double cross hybrid prediction in maize when epistasis is present. Crop Science, Madison, v. 4, n. 4, p. 363-366, 1964

FEASTER, C. V.; TURCOTTE, E. L. Yield stability in doubled haploids of American pima cotton. Crop Science, Madison, v. 13, n. 2, p. 232-233, 1973.

HALLAUER, A. R. Development of single-cross hybrids from two eared maize populations. Crop Science, Madison, v. 7, p. 192-195, 1967.

LEITE, A. C. S. Adaptabilidade, estabilidade, heterose e avaliação de metodologias alternativas de seleção recorrente recíproca, com famílias de irmãos completos, em cultivos de milho (Zea mays L.). Viçosa : UFV, 1988 121 p. Tese de Doutorado.

LEMOS, M. A. Variabilidade fenotípica em híbridos simples, híbridos duplos, variedades e compostos de milho. Piracicaba : ESALQ, 1976. 62 p. Dissertação de Mestrado.

LIN, C. S.; BINNS, M. R. A superiority measure of cultivar performance for cultivar $\mathrm{x}$ lacation data. Canadian Journal of Plant Science, Ottawa, v. 68, n. 1, p. 193-198, 1988.

LONNQUIST, J. H.; WILLIAMS, N. E. Development of maize hybrids through selection among full sib families. Crop Science, Madison, v. 7, p. 369-370, 1967.
LOPES, M. T. G. Capacidade produtiva, adaptabilidade, e estabilidade de híbridos de famílias endogâmicas de milho (Zea mays L.), obtidos pelo método dos híbridos crípticos. Viçosa : UFV, 1999. 96 p. Dissertação de Mestrado.

NASPOLINI FILHO, V. Variabilidade fenotípica e estabilidade em híbridos simples, híbridos duplos, variedades e compostos de milho. Piracicaba : ESALQ, 1976. 68 p. Dissertação de Mestrado.

PATERNIANI, E. Seleção recorrente para capacidade geral de combinação em milhos da América Central. Ciência e Cultura, São Paulo, v. 17, n. 4, p. 555-559, 1965

PIMENTEL-GOMES, F. Curso de Estatística Experimental. 11. ed. Piracicaba : Nobel, 1987. 466 p

RUSCHEL, R. Interação genótipo $\mathbf{x}$ localidades na região Centro-Sul em milho (Zea mays $\mathbf{L}$.). Piracicaba : ESALQ, 1986. 60 p. Dissertação de Mestrado

RUSCHEL, R.; PENTEADO, F. Análises dos componentes da variância de duas classes de cultivares de milho e estimativa do progresso genético médio em ensaios de produção. Pesquisa Agropecuária Brasileira, Rio de Janeiro, v. 5, n. 3, p. 381-388, 1970.

SPRAGUE, G. F. Corn breeding. In: CORN AND CORN IMPROVEMENT. New York : Academic, 1955. p. 221-292 